\title{
Загадка парадеигмы
}

\author{
Наталья Валентиновна Зайцева \\ Вероссийская академия внешней торговли. \\ Российская Федерация, 119285, г. Москва, ул. Пудовкина, д. 4А. \\ E-mail: natvalen@list.ru
}

Аннотация: Статья продолжает исследование парадеигмы, или рассуждения на основании примера. Это рассуждение анализируется Аристотелем в «Первой Аналитике», и в риторическом ключе рассматривается как один из способов убеждения - в «Риторике». В предыдущих статьях акцент был сделан на когнитивно-эпистемологической характеристике соответствующей познавательной процедуры, в данной работе в центре внимания оказываются логические характеристики парадеигмы. В первом разделе анализируются соответствующие фрагменты текста Аристотеля и дается краткое изложение когнитивно-феноменологического анализа парадеигмы, устанавливается ее связь с аналогизирующей апперцепцией (аппрезентацией) Гуссерля. В следующем разделе выявляется логическая форма рассуждения на основании примера, показывается его несводимость к другим типам правдоподобных (недедуктивных) рассуждений, таким как обобщающая индукция, аналогия и абдукция. На этом основании выдвигается предположение о том, что прадаеигма представляет собой особый самостоятельный вид правдоподобных рассуждений. В заключительной части статьи рассматривается роль парадеигмы и лежащей в ее основе когнитивной процедуры в логико-философских взглядах Аристотеля. Особое внимание уделяется соответствующей когнитивной процедуре познания первоначал, описываемой Аристотелем во «Второй Аналитике».

Ключевые слова: парадеигма, правдоподобные рассуждения, Аристотель, универсальные когнитивные механизмы рассуждений

Для цитирования: Зайцева Н.В. Загадка парадеигмы // Логические исследования / Logical Investigations. 2019. T. 25. № 1. C. 37-51. DOI: 10.21146/2074-1472-2019-25-1-37-51

\section{Введение}

Строго говоря, с парадеигмой (paradeigma) как особого рода риторическим рассуждением и лежащей в его основе когнитивной процедурой связана не одна, а сразу несколько загадок. Аристотель обращается к этому рассуждению несколько раз, наиболее известные фрагменты содержатся в «Риторике» и «Первой Аналитике» (см. Аристотель, 1978]). В «Риторике» Аристотель, 2005 рассуждение на основании примера (известное также как «пример», «параллельное рассуждение» и «парадеигма», 
paradeigma) выделяется в качестве одного из двух способов убеждения наряду с риторическим силлогизмом (энтимемой) Аристотель, 2005, 1356b5]. В связи с этим возникает первый вопрос и, соответственно, первая загадочная характеристика парадеигмы: что делает рассуждение на основании примера настолько убедительным, что заставляет Аристотеля придавать ему в процессе аргументации не меньшее значение, чем силлогизму? Следующий вопрос непосредственно связан с первым: какую роль играет рассуждение на основании примера и соответствующая когнитивная операция в логико-философской теории Аристотеля? Наконец, что же представляет собой парадеигма как рассуждение? Какова ее логическая форма, какое место следует ей отвести в современной классификации рассуждений?

Данная статья продолжает мои исследования последних лет. В предыдущих работах я стремилась в первую очередь дать ответ на первый из поставленных вопросов. Эти размышления привели меня к усмотрению определенного соответствия между когнитивной процедурой, лежащей в основе парадеигматического рассуждения, и аналогизирующей апперцепцией (аппрезентацией) Э. Гуссерля. Достаточно подробно обоснование этого соответствия приводится в работе Зайцева, 2018. В настоящей статье я хотела бы более подробно рассмотреть парадеигму с логической точки зрения и в определенной степени коснуться ее роли для логико-философской концепции Аристотеля в целом.

Структура работы такова. В следующем параграфе я приведу характеристику парадеигмы Аристотелем и кратко, для придания статье самодостаточности, охарактеризую когнитивные основания этого рассуждения. Во втором параграфе будет предложена реконструкция парадеигмы и рассмотрен вопрос о типе этого рассуждения. В заключительной части будет предпринята попытка обозначить ту роль, которую отводит парадеигме Аристотель в своих силлогистических построениях и более широкой логико-философской концепции.

\section{1. Парадеигма Аристотеля и аппрезентация Гуссерля}

Рассуждение на основании примера, как было отмечено выше, рассматривается Аристотелем в «Риторике» как способ убеждения, а в «Первой Аналитике» - в контексте силлогистических рассуждений. В принципе соответствующие фрагменты текстуально очень близки, но в «Первой Аналитике» изложение чуть более подробное и ясное, поэтому далее я преимущественно буду опираться на этот текст. Непосредственно парадеигме посвящена 24 глава 2 книги «Первой Аналитики» Аристотель, 1978, Первая аналитика, II, 24, 68b38 - 69a19]. 
Согласно Аристотелю, «пример приводится, когда доказывается, что [больший] крайний термин присущ среднему через подобие третьему. При этом должно быть известно, что средний термин присущ третьему, а первый - тому, что подобно третьему» Аристотель, 1978, Первая аналитика, II, 24, 68b40]. Далее вводятся обозначения, и в целом следуя Аристотелю, позволю себе для удобства восприятия его идеи использовать строчные буквы для обозначения единичных терминов. Пусть А обозначает «зло», Б - «начинать войну с соседями», b - «война афинян с фиванцами», d «война фиванцев с фокейцами». «Итак, если мы хотим доказать, что вести войну с фиванцами есть зло, то нужно принять, что вести войну с соседями есть зло. Но это становится убедительным из [наблюдения] подобных случаев, например из того, что для фиванцев война с фокейцами есть зло. И так как война с соседями есть зло, а война с фиванцами есть война с соседями, то очевидно, что вести войну с фиванцами есть зло. Поэтому очевидно, что Б присуще b и d (ибо и то, и другое есть ведение войны с соседями). Также очевидно, что А присуще d (ибо фиванцам война с фокейцами не принесла добра); а что А присуще b - это будет доказано через d» Аристотель, 1978, Первая аналитика, II, 24, 69а5-10].

Предварительный анализ приведенного фрагмента позволяет выявить следующее. Во-первых, парадеигма подразумевает обоснование большей посылки силлогизма. Во-вторых, делается это через подобие двух (или более) случаев. В приведенном примере таковыми случаями являются две войны - афинян с фиванцами (b) и фиванцев с фокейцами (d). Кроме того, для обоснования общего высказывания (большей посылки последующего силлогизма) «Война с фиванцами есть зло» используется еще одна посылка - единичное утверждение «Война фиванцев с фокейцами есть зло» (d есть $\mathrm{A})$. Таким образом, использование рассуждения на основании примера предполагает два этапа - простой категорический силлогизм и предшествующее ему рассуждение на основании подобия. Условимся именно последнее рассуждение называть парадеигмой в узком смысле.

Далее Аристотель останавливается на специфике парадеигмы. «Таким образом, очевидно, что пример показывает отношение не части к целому и не целого к части, а отношение части к части, когда и та и другая подчинены одному и тому же, но одна из них известна» Аристотель, 1978, Первая аналитика, II, 24, 69a15]. Аналогичные фрагменты в «Риторике» звучат чуть по-другому, возможно в силу особенностей перевода. «Пример не выражает ни отношения части к целому, ни целого к части, ни целого к целому, но части к части, подобного к подобному, когда оба случая относятся к одному роду, причем один более известен, чем другой» Аристотель, 2005. Риторика, 1357b30]. В другом переводе «Риторики» (издательство 
«Азбука», Санкт-Петербург, 2000 г.) вместо «относятся к одному роду» использована конструкция «оба случая подходят под одну и ту же категорию случаев».

Во второй книге «Риторики» пример рассматривается уже в более практической, аргументативной плоскости. Здесь Аристотель делает важное различение, на которое, в частности, обращает внимание автор |McCormic, 2014. Стагирит не просто различает два вида примеров - сообщение о реальном событии и о вымышленном, каковым является притча или басня, - он использует для них разные термины: paradeigma в первом случае и parabole во втором [Аристотель, 2005, Риторика, II, 1393a30]. Таким образом, парадеигма предполагает апелляцию к реальным событиям, а подобие (или отождествление) двух случаев основано на их отнесении к общему роду, или категории.

Последнее соображение интересно соотнести с изложенной в 16-й главе 2 -й книги «Первой аналитики» процедурой постулирования оснований. Начинается глава с характеристики постулирования. «Постулирование и принятие начала по роду своему относится к [рассуждению], не доказывающему предложенного...» Аристотель, 1978, Первая аналитика, II, 16, 64b30]. Проясняя специфику этой процедуры, Аристотель отмечает, что «начала познаются через самих себя» (см. [Аристотель, 1978, Там же, 64b35]), это «значит постулировать начало, это мы сказали выше, а именно: доказывать то, что не самоочевидно, через него же (а это значит не доказывать)». Трактовка этой процедуры как доказательства приводит к кругу в рассуждениях ([Аристотель, 1978, Там же, 65a1-10]). Постулирование существенным образом основано на тождестве терминов (и соответствующих случаев): «тождественные [термины] присущи одному и тому же, или потому, что одно и то же присуще этим [терминам]...» Аристотель, 1978, Там же, 65a30]. При этом постулирование должно давать достоверное знание: «Постулирование начала в доказательствах касается того, с чем дело действительно обстоит так-то и так-то» Аристотель, 1978, Там же, 65а35].

Итак, отождествление двух случаев - нового, относительно которого выносится суждение, и реального прецедента, или образца - играет важную роль в парадеигматическом рассуждении. Недаром одно из значений слова «парадеигма» (парадигма) - «модель или образец».

Как уже отмечалось выше, на мой взгляд, когнитивные основания рассуждения на основании примера могут быть адекватно реконструированы в терминах аппрезентации (аналогизирующей апперцепции), рассмотренной Э. Гуссерлем в «Картезианских размышлениях». Механизм аппрезентации, по Гуссерлю, обеспечивает типизацию объектов окружающего мира через перенос смысловых характеристик с предмета-образца на новый 
объект. При этом Гуссерль настаивает на том, что аппрезентация не является рассуждением. «Апперцепция не есть вывод, не есть мыслительный акт. Каждая апперцепция, в которой мы с одного взгляда воспринимаем и, фиксируя свое внимание, схватываем заранее данные предметы (к примеру, заранее данный повседневный мир), каждая апперцепция, в которой мы сразу же понимаем их смысл вместе с его горизонтами, интенционально отсылает нас к некому первичному учредительному акту, когда был впервые конституирован предмет, обладающий подобным смыслом» Гуссерль, 1998 , с. 217].

Суть этой процедуры представлена в 51-м параграфе «Картезианских рассуждений» «Удвоение как ассоциативно конституирующий компонент опыта другого». Удвоение представляет собой изначальную форму пассивного синтеза. Два предмета (образец и объект-стимул) даны интенционально как пара, смысловые характеристики членов этой пары перекрываются, что ведет к взаимному перенесению смысла. В результате смысл переносится с образца на новый объект, при этом каждый новый объект в процессе типизации обогащает хранящийся в памяти образец.

Возвращаясь к трактовке парадеигмы, вполне оправданно трактовать подобие двух случаев не как некоторое симметричное отношение, а как отождествление этих случаев на основе распознания в новом объекте стороны или части объекта-образца, пережитого ранее. Скажем, в аристотелевском примере две войны $\mathrm{b}$ и $\mathrm{d}$ отождествляются как относящиеся $\mathrm{k}$ категории Б «война с соседями». Это приводит к пониманию, что именно война с соседями есть зло, что обеспечило негативные последствия (стало их причиной) упомянутой войны фиванцев с фокейцами (d).

Таким образом, как это было отмечено в статье [Зайцева, 2018, с. 21], «когнитивная процедура аппрезентации, включающая (а) удвоение и собственно (b) аппрезентативный перенос на уровне рассуждения, предстает как (a) риторический пример, (b) сопровождающийся для каждого конкретного случая соответствующим силлогизмом».

\section{2. Парадеигма как правдоподобное рассуждение}

Аристотель достаточно четко и ясно описывает парадеигму. Более того, в «Риторике» он дает ее обобщенную характристику. K сожалению, русский перевод этого фрагмента не отличается ясностью, в то время как стандартный английский перевод буквально содержит описание логической формы парадеигматического рассуждения. «Энтимемы, основанные на примере, это те, которые ведут от одного или более сходных случаев к общему суждению, а затем обосновывают дедуктивно единичное заключение» Аристотель, 2005, Риторика, 1402b15] (перевод на русский язык вы- 
полнен по (Kennedy, 1991]). Все это позволяет выделить логическую форму этого рассуждения (в узком смысле) следующим образом:

d M-подобно b, d есть Р, следовательно, все М есть Р.

В рассмотренном выше примере на основании подобия двух войн в том, что обе они - войны с соседями, и утверждения, что одна из них есть зло, делается заключение о том, что всякая война с соседями есть зло. Таким образом, из единичного высказывания и особого рода высказывания о подобии относительно некоторой категории выводится общеутвердительное заключение. Очевидно, что подобное рассуждение нельзя считать дедуктивным. K какому же типу правдоподобных рассуждений можно отнести парадеигму?

Наиболее типичные варианты ответа - это (обобщающая) индукция или аналогия. Рассмотрим их по порядку.

Трактовка парадеигмы как индуктивного рассуждения (наведения) коренится в замечаниях самого Аристотеля по этому поводу. Так, в «Риторике» говорится: «пример есть индукция» (термин epagôgê у Аристотеля переводится и как «наведение», и как «индукция») Аристотель, 2005, Риторика, 1356b], во «Второй Аналитике» находим, что убеждение осуществляется «посредством примеров, которые суть наведения» Аристотель, 1978, Вторая аналитика, I, 1, 71а10].

Такое понимание рассуждения на основе примера имеет долгую историю. Характерный пример представляет собой работа [Benoit, 1980, в заглавие которой прямо вынесено утверждение о том, что парадеигма есть риторическая индукция. Автор достаточно подробно рассматривает различные интерпретации рассуждения на основе примера, стремясь обосновать вывод о его индуктивном характере. Однако, даже приходя к такому заключению, автор называет рассуждение на основе примера особой «риторической индукцией», отмечая ее бросающиеся в глаза отличия от стандартного индуктивного обобщения. В более современных исследованиях, например в статье о риторике Аристотеля в Стэнфордской Энциклопедии философии, также констатируется индуктивный характер парадеигмы, правда, без подробного обоснования.

Сам Аристотель, с одной стороны, свидетельствует в пользу такого понимания, с другой стороны, приводит аргументы против индуктивной трактовки парадеигмы. Так, в той же «Риторике» он пишет: «Пимерами следует пользоваться после энтимем в виде эпилога, тогда как в начале они похожи на индукцию, а ораторским речам индукция не свойственна» Аристотель, 2005, Риторика, 1394а10]. Если примеры всего лишь «похожи на индукцию», значит, они ею не являются? Примерно также обстоит 
дело с парадеигмой и в «Аналитиках». Упоминавшуюся выше 24-ю главу 2-й книги «Первой Аналитики», посвященную анализу рассуждения на основе примера, Аристотель завершает таким выводом: «От наведения пример отличается тем, что наведение доказывает присущность [большего] крайнего термина среднему из всех единичных [случаев] и не умозаключает относительно [меньшего] крайнего термина, пример же умозаключает относительно меньшего термина и доказывает не из всех [единичных случаев]» Аристотель, 1978, Первая аналитика, II, 24, 69a16-19].

Как мне представляется, источником некоторой путаницы служат не различия в переводах, а не вполне однозначное изложение мыслей Аристотеля в его же собственных работах. Хорошо известна непростая судьба его рукописей и набросков, обретавших новую жизнь в интерпретации учеников и последователей. Относительно трактовок парадеигмы можно с определенными основаниями предположить, что для Аристотеля, рассматривающего силлогизм и индукцию (наведение) как основные типы рассуждений, энтимема и пример (парадеигма) становятся основными типами убеждения. При этом энтимема представляет собой риторический аналог силлогизма, а пример - такой же аналог наведения, но в сфере риторики.

Во многом ответ на вопрос, является ли парадеигма индуктивным рассуждением, зависит от того, как понимается индукция. Если, как это часто бывает, индуктивные рассуждения понимаются как недедуктивные, правдоподобные, то рассуждение на основе примера должно быть отнесено к таковым. Если же придерживаться более узкого понимания индукции, то с подобной оценкой парадеигмы согласиться уже нельзя. Этому мешают две ее особенности: наличие одной единичной посылки (в принципе таких посылок может быть и больше, но Аристотель прямо указывает, что достаточно и одной) и наличие особой посылки, в которой утверждается подобие двух случаев относительно некоторой категории. Резюмируя, можно сказать, что и в обобщающей индукции, и в индукции «к следующему за», и в исключающей индукции, во-первых, вывод делается не на основании единственного примера, а во-вторых, не используется утверждение о подобии каких-то случаев.

В таком случае, возможно, следует понимать парадеигму как рассуждение по аналогии? Последнее как раз предполагает апелляцию к подобию двух предметов (или систем предметов) и использование в качестве посылки единичного высказывания об одном из них. Такая интерпретация Аристотеля встречается довольно часто. В качестве примера сошлюсь на работу [Contreras, 2015], а также на принятую в российской традиции интерпретацию, восходящую к трудам Маковельского (Маковельский, 2004 
и Ахманова Ахманов, 2002 и продолжающуюся в более современных работах (см., например, |Вольф, 2013]).

Следует отметить, что сам Аристотель использует термин «аналогия» (analogia) в близком к Платону математическом смысле, характеризующем математическую пропорцию, но не рассуждения. Рассуждения по аналогии Стагирит описывает, используя термин «сходство» (homoiotes). Наиболее характерные фрагменты содержатся в «Топике» Аристотель, 1978, Топика I, 17 и 18, VII, 1]). «Далее следует выведывать на основании сходства, ибо это убедительно и лучше скрывает общее... Хотя этот [прием] сходен с наведением, однако не тождественен ему: в наведении общее принимается на основании единичного, а при указании сходства не получается общее, охватывающее все случаи сходства» Аристотель, 1978, Топика, VIII, 1, 156b10-17]. И в самом деле, аналогия приводит к единичному заключению, в то время как заключение парадеигмы (в узком смысле) - это общее высказывание. Если же пытаться трактовать парадеигму в широком смысле (состоящую из двух этапов) как рассуждение по аналогии, то тут, напротив, излишним оказывается дедуктивный, силлогистический этап. Таким образом, остается согласиться с автором [Bartha, 2010], замечающим, что рассуждение на основании сходства куда ближе к нашему современному пониманию аналогии, чем парадеигма.

Куда интереснее, на мой взгляд, сравнить парадеигму и абдукцию. На первый взгляд поиск объясняющей гипотезы (inference to the best explanation) куда ближе к рассуждению на основании примера, чем индукция или аналогия. Термин «абдукция» был введен, как известно, Чарльзом Пирсом. Его понимание абдукции претерпевало трансформации. Наиболее известны следующие варианты.

1. Имеется правило, например, «Все бобы из этой корзины - белые», и результат: «Эти бобы белые». Следовательно, «эти бобы из этой корзины».

2. Наблюдается любопытный факт $C$. Если бы утверждение $A$ было истинным, $C$ было бы само собой разумеющимся. Следовательно, есть основания предполагать, что $A$ истинно.

В современных исследованиях в рамках парадигмы искусственного интеллекта абдукция обобщается до следующей схемы рассуждений:

$$
C, A \longrightarrow C / A \text { Aliseda, 2017. }
$$

Иногда выделяют множество разновидностей абдуктивных рассуждений, включая в них и аналогию, и парадеигму. Порой, следуя традиции 
Пирса, разделяют все рассуждения на дедуктивные, индуктивные и абдуктивные. При таком подходе, наверное, и в самом деле остается считать парадеигму абдуктивным рассуждением, поскольку, как было показано выше, она не относится ни к дедуктивным, ни к индуктивным. Однако если все-таки рассматривать абдукцию не в расширительной трактовке, то принципиальное, на мой взгляд, отличие ее от парадеигмы состоит в следующем. Задача абдукции - предложить объясняющую гипотезу. При этом сама процедура объяснения трактуется в соответствии с дедуктивнономологической моделью Гемпеля [Aliseda, 2017, p. 222]. Таким образом, абдукция предполагает апелляцию к некоторому законоподобному утверждению, а смысл объясняющей гипотезы состоит в том, что это общее высказывание расширяется так, чтобы включать и те самые любопытные факты. По сути дела, выдвижение такой гипотезы сводится к расширению экстенсионала субъекта общего высказывания. В случае парадеигмы мы получаем абсолютно новое знание без использования каких-то общих высказываний, в противном случае процедуру обоснования больших общеутвердительных посылок силлогизма можно было бы свести к дедуктивному (силлогистическому) получению одних общих высказываний из других, что явно не соответствовало бы интенции Аристотеля.

Возвращаясь к Аристотелю, интересно заметить, что еще до Пирса термин abductio был введен Джулио Пейсом (Julius Pacius) как латинский перевод термина apagoge, использованного Аристотелем для характеристики особого типа рассуждения. Этот тип рассуждений был рассмотрен в Первой Аналитике после описания парадеигмы - глава 24 «Доказательство посредством отведения Аристотель, 1978, Первая аналитика, II, 25, 69a20-35]. На это обращают внимание авторы программной статьи Gabbay, Woods, 2006. Таким образом, если вести историю выделения абдуктивных рассуждений от Аристотеля, то эти рассуждения принципиально отличаются от парадеигмы.

Итак, рассуждение на основании примера не может быть отнесено ни к индуктивным, ни к абдуктивным рассуждениям, ни к рассуждениям по аналогии. На мой взгляд, парадеигму нельзя в принципе отнести к известным разновидностям правдоподобных рассуждений. Это означает, что в этом случае мы имеем дело с особым типом недедуктивного рассуждения.

\section{Заключение. Парадеигма в логико-философской теории Аристотеля}

Можно предположить, что парадеигма играет в логико-философских построениях Аристотеля двойную роль, что связано с ее разными ипостасями. Во-первых, рассуждение на основании примера играет определен- 
ную роль в силлогистической теории Аристотеля, во-вторых, оно позволяет обнаружить фундаментальный когнитивный механизм, лежащий в основе этого рассуждения.

Как известно (см. [Бочаров, Маркин, 2013]), силогистика Аристотеля представляет собой особую самостоятельную силлогистическую теорию. В ней неверны законы силлогистического тождества, принимаются не все принципы превращения, верные в традиционной силлогистике, и т. п. Представляется, что рассмотренное выше парадеигматическое рассуждение в определенном смысле может прояснить специфику аристотелевской силлогистики.

Вернемся к логической форме парадеигмы в узком смысле. Одной из посылок является утверждение о подобии двух случаев относительно некоторой категории (свойства). Для удобства записи условимся обозначать логическую форму высказывания вида « $a$ подобно $b$ относительно $P \gg$ как $a={ }_{P} b$, а для логической формы общеутвердительного высказывания использовать стандартную нотацию - SaP. Довольно естественно предположить, что условием для самотождественности произвольного объекта относительно некоторого свойства является его присущность данному объекту, выражаемая единичным высказыванием. Последнее можно выразить как своеобразный «Принцип самоподобия»: если $a$ есть $P$, то $a={ }_{P} a$.

Принятие этого самоочевидного принципа позволяет построить следующее рассуждение:

1. $c$ есть $P$

2. $c={ }_{P} c$

3. $с$ есть $P$ и $c={ }_{P} c$

4. $P a P$

5. если $c$ есть $P$, то $P$ aP
- допущение

- из 1, по Принципу самоподобия

- из 1 и 2

- из 3, по парадеигме

- из 4, введением импликации

Таким образом, необходимым условием для закона тождества оказывается непустота термина, выражаемая единичным высказыванием о принадлежности предмету соответствующего признака.

В принципе возможно построение теории силлогистического типа с двумя дополнительными дедуктивными постулатами: парадеигмой и принципом самоподобия. В такой теории производным мог бы оказаться принцип введения общеотрицательных высказываний: $a={ }_{S} b, a$ есть не- $P$, следовательно, $S e P$ и т. п.

Вторая роль парадеигмы в большей степени связана с лежащей в ее основе когнитивной процедурой. В завершающей «Вторую Аналитику» 19й главе 2-й книги, озаглавленной «Познание начал», Аристотель фактически обращается все к той же проблеме обоснования общих высказываний. 
Но теперь он не останавливается на обосновании как разновидности доказательства или рассуждения, поскольку выше (в 16-й главе 2-й книги «Первой аналитики») эта проблема уже обсуждалась, говоря современным языком, теперь Аристотеля волнует когнитивная составляющая такого обоснования. И эту когнитивную основу он видит в особой способности: «Поэтому необходимо обладать некоторой способностью [познания] (dynamis)... Но такая способность, очевидно, присуща всем животным, ибо они обладают прирожденной способностью различать, которая называется чувственным восприятием» Аристотель, 1978, Вторая аналитика, II, 19, 99b30-35]. Далее Аристотель поясняет, что имеется в виду под способностью различать: «Другие же, когда они воспринимают чувствами, что-то удерживают в душе. Если же таких [запечатлений] много, то возникает уже некоторое различие, так что из того, что остается от воспринятого, у одних возникает некоторое понимание, а у других нет» Аристотель, 1978. Там же, 99b39 - 100a3]. И далее, «из единого, отличного от множества, того единого, что содержится как тождественное во всем этом множестве, берут свое начало искусство и наука» Аристотель, 1978, Там же, 100а10]. Осознавая некоторую расплывчатость приведенных объяснений, Аристотель видит необходимость объяснить еще раз: «В самом деле, если одно, не отличающееся от другого, удерживается, то появляется в душе первое общее (ибо хотя воспринимается единичное, но восприятие есть [восприятие] общего, например человека, а не человека Каллия). Снова останавливаются на этом, пока не удерживается нечто неделимое и общее, например, вместо живого существа такого-то [вида] - живое существо [вообще], и далее таким же образом» Аристотель, 1978, Там же, 100a15 - 100b5].

Таким образом, убедительность парадеигмы как риторического приема основана на фундаментальной когнитивной способности, которую, в частности, пытался описать Гуссерль через механизм аппрезентации, основанный на генеративном a priori - интенции к отождествлению. Эта способность является не только фундаментальной, но и универсальной, присущей не только человеку, но и другим животным. Аппрезентативный перенос смысла с объекта образца на новый объект, осуществляемый на основании отождествления, оказывается у Гуссерля необходимым условием познания, позволяющим избежать бесконечного разнообразия мира. Аналогично парадеигма Аристотеля может рассматриваться как попытка усмотрения и вербализации первоначал. Выражая универсальный первоначальный принцип познания, легитимизируя операцию наделения смыслом любого объекта на основании пережитого ранее даже единичного случая, парадеигма в узком смысле не может быть отнесена ни к одному из существующих типов рассуждений. Она является условием этих рассуждений, что, собственно, 
подтверждается Аристотелем, когда он рассматривает рассуждение на основе примера как обосновывающее общую посылку силлогизма.

Фундаментальность и универсальность когнитивной парадеигмы проявляется в том, что подобное рассуждение присутствует в различных системах мышления, основанных на разных понятиях рациональности. В качестве примера можно обратиться к статьям А.В. Смирнова Смирнов, 2017 и Смирнов, 2018, в которых анализируется особое рассуждение «кийассоизмерение» (qiyas). В частности, автор стремится показать, что это рассуждение не является рассуждением по аналогии, как считают большинство комментаторов. «Кийас-соизмерение», так же как и парадеигма, основано на подобии, при этом подобное понимается как наиболее близкое (соизмеримое). «Так устанавливается, что нечто схоже с чем-то другим, причем эта схожесть фиксируется как общность атрибута, как отнесение к общему классу» Смирнов, 2017, с. 83]. При этом нечто обладает «смыслом», подобным «смыслу» исходного случая» Смирнов, 2017, с. 84]. Примечательно, что на сходство парадеигмы Аристотеля и особого, «обосновывающего случая «кийас-соизмерения» (qiyas al-illa) обращают внимание и авторы работы |Rahman, Iqbal, 2018]: «Крайне важно отметить, что метод, используемый кийас-аль-илла-формой ко-реляционного вывода, нацелен не на установление сходства между источником и вариацией. Цель состоит в том, чтобы обнаружить общий закон и свойство, общее для обоих случаев, что позволяет вывести некоторое лежащее в основании правило. На самом деле это не пример аналогии сходств, а то, что в наше время называют дедуктивным параллельным рассуждением».

Таким образом, основанная на фундаментальном, универсальном, встроенном (телесно воплощенном) когнитивном механизме, парадеигма представляет собой не просто один из способов риторического убеждения, но и может рассматриваться как вербальное выражение познавательной способности, обеспечивающей саму возможность усмотрения закономерностей в мире.

\section{Литература}

Аристотель, 1978 - Apистотель. Соч.: в 4 т. Т. 2. М.: Мысль, 1978. 687 с.

Аристотель, 2005 - Аристотель. Риторика. Поэтика. М.: Лабиринт, 2005. 256 с.

Ахманов, 2002 - Ахманов А.С. Логическое учение Аристотеля. 2-е изд., стереотип. М.: Едиториал УРСС, 2002. 316 с.

Бочаров, Маркин, 2013 - Бочаров В.А., Маркин В.И. Силлогистические теории. M.: Directmedia, 2013. 333 c. 
Вольф, 2013 - Воль М.Н. Основания аргументации в раннегреческой философии: аналогия как тип аргументации // Вестник НГУ. Философия. 2013. Т. 11. № 2. C. $110-119$.

Гуссерль, 1998 - Гуссерль Э. Картезианские размышления. СПб.: Наука: Ювента, 1998. 315 c.

Зайцева, 2018 - Зайцева Н.В. Когнитивно-феноменологическая интерпретация риторического примера // Вестник Томского государственного университета. Философия. Социология. Политология. 2018. № 45. С. 14-24.

Маковельский, 2004 - Маковельский А.О. История логики. Жуковский; М.: Кучково поле, 2004. $480 \mathrm{c.}$

Смирнов, 2017 - Смирнов А.В. О формализации умозаключения в процессуальной логике. Часть I // Философский журнал. 2017. Т. 10. № 4. С. 71-92.

Смирнов, 2018 - Смирнов А.В. О формализации умозаключения в процессуальной логике. Часть II // Философский журнал. 2018. Т 11. № 1. С. 5-27.

Aliseda, 2017 - Aliseda A. The Logic of Abduction: An Introduction // Springer Handbook of Model-Based Science / Ed. by L. Magnani, T. Bertolotti. Springer Cham., 2017. P. 219-230.

Bartha, 2010 - Bartha P. By parallel reasoning. Oxford University Press, 2010. 356 p.

Benoit, 1980 - Benoit W.L. Aristotle's example: The rhetorical induction // Quarterly Journal of Speech. 1980. Vol. 66. No. 2. P. 182-192.

Contreras, 2015 - Contreras A.R. Analogy and Isomorphism: Philosophy, Mathemafics and Space // 1st World Congree on Analogy. Puebla, Mexico. 2015. 62 p.

Gabbay, Woods, 2006 - Gabbay D., Woods J. Advice on abductive logic // Logic Journal of IGPL. 2006. Vol. 14. No. 2. P. 189-219.

Kennedy, 1991 - Kennedy G.A. (trans./ed.). Aristotle 'On Rhetoric': A Theory of Civic Discourse. New York/Oxford: Oxford University Press, 1991. 337 p.

McCormic, $2014-$ McCormick S. Argument by comparison: An ancient typology // Rhetorica: A Journal of the History of Rhetoric. 2014. Vol. 32. No. 2. P. $148-164$.

Rahman, Iqbal, 2018 - Rahman S., Iqbal M..Unfolding Parallel reasoning in islamic jurisprudence: Epistemic and Dialectical Meaning in Abu Ishaq al-Shirazi's System of Co-Relational Inferences of the Occasioning Factor // Arabic Sciences and Philosophy. Vol. 28. No. 1. P. 67-132. 


\title{
Natalia V. Zaitseva \\ The riddle of paradeigma
}

\author{
Natalia V. Zaitseva \\ Russian Foreign Trade Acdemy, \\ 4a Pudovkin St., Moscow, 119285, Russian Federation. \\ E-mail: natvalen@list.ru
}

\begin{abstract}
The article continues the study of paradeigma (parallel reasoning, reasoning based on an example). Paradeigma was considered in detail by Aristotle in Prior Analytics, and in a rhetorical manner - in "Rhetoric" as being one of the two modes of argument. In previous papers, the emphasis was made on cognitive-epistemological interpretation of corresponding cognitive procedure. This paper zeros on the logical characteristics of paradeigma. The first section contains the analysis of the relevant fragments of the Aristotle's text and brief summary of the cognitive-phenomenological interpretation of paradeigma. The aim of the next section was to identify the logical form of reasoning based on an example. It is shown to be non-reducible to other types of plausible (non-deductive) reasoning, such as inductive generalization, analogy and abduction. On this basis, a reasonable assumption is made that pradaeigma is a special independent kind of plausible reasoning. In the final part of the article, the place of the paradeigma and the underlying cognitive procedure in the logical and philosophical views of Aristotle is considered. Special attention is paid to the corresponding cognitive procedure of the first principle grasping, as described by Aristotle in the Posterior Analytics.
\end{abstract}

Keywords: paradeigma, plausible reasoning, Aristotle, universal cognitive mechanisms of reasoning

For citation: Zaitseva N.V. "Zagadka paradeigmy" [The riddle of paradeigma], Logicheskie Issledovaniya / Logical Investigations, 2019, Vol. 25, No. 1, pp. 37-51. DOI: 10.21146/20741472-2019-25-1-37-51 (In Russian)

\section{References}

Akhmanov, 2002 - Akhmanov, A. C. Logicheskoe uchenie Aristotelya [Aristotle's Logical Doctrine]. (2nd ed.). Moscow: Editorial URSS, 2002. 316 pp.(In Russian)

Aliseda, 2017 - Aliseda, A. "The Logic of Abduction: An Introduction", Springer Handbook of Model-Based Science, ed. by L. Magnani, T. Bertolotti. Springer Cham., 2017, pp. 219-230.

Aristotle, 1978 - Aristotel'. Sochineniya $v 4$ tomakh [Writings in 4 volumes]. Vol 2. Moscow: Mysl, 1978. 687 pp.(In Russian)

Aristotle, 2005 - Aristotel'. Ritorika. Poetika. [Rhetoric. Poetics]. Moscow : LabirintPress, 2005. 256 pp.(In Russian) 
Bartha, 2010 - Bartha, P. By Parallel Reasoning. Oxford University Press, 2010. $356 \mathrm{pp}$.

Benoit, 1980 - Benoit, W.L. "Aristotle's Example: The Rhetorical Induction", Quarterly Journal of Speech, 1980, Vol. 66, No. 2, pp. 182-192.

Bocharov, Markin, 2013 - Bocharov, V.A., Markin, V.I. Sillogisticheskie teorii. [Syllogistic Theories]. Moscow: Directmedia, 2013, 333 pp. (In Russian)

Contreras, 2015 - Contreras, A.R. "Analogy and Isomorphism: Philosophy, Mathemafics and Space", in: Proc. 1st World Congree on Analogy. Puebla, Mexico, 2015, $62 \mathrm{pp}$.

Gabbay, Woods, 2006 - Gabbay, D., Woods, J. "Advice on Abductive Logic", Logic Journal of IGPL, 2006, Vol. 14, No. 2, pp. 189-219.

Husserl, 1998 - Gusserl', E. Kartezianskie razmyshleniya. [Cartesian Meditations]. St. Petersburg: Nauka: Juventas, 1998, 315 pp. (In Russian)

Kennedy, 1991 - Kennedy, G.A. (trans./ed.). Aristotle 'On Rhetoric': A Theory of Civic Discourse. New York/Oxford: Oxford University Press, 1991. 337 pp.

Makovel'skii, 2004 - Makovel'skii, A.O. Istoriya logiki. [History of Logic]. Joukowski; Moscow: Kuchkovo pole, 2004. 480 pp. (In Russian)

McCormic, 2014 - McCormick, S. "Argument by Comparison: An Ancient Typology", Rhetorica: A Journal of the History of Rhetoric, 2014, Vol. 32, No. 2, pp. $148-164$.

Rahman, Iqbal, 2018 - Rahman, S., Iqbal, M. "Unfolding Parallel Reasoning in Islamic Jurisprudence: Epistemic and Dialectical Meaning in Abu Ishaq al-Shirazi's System of Co-Relational Inferences of the Occasioning Factor", Arabic Sciences and Philosophy, Vol. 28, No. 1, pp. 67-132.

Smirnov, 2017 - Smirnov, A.V. O formalizatsii umozaklyucheniya v protsessual'noi logike. Chast' I [Qiyās as a Formal Proof: the Way the Fuqahā' Argued. Part I], Filosofskii zhurnal [Philosophy Journal], 2017, Vol. 10, No. 4, pp. 71-92. (In Russian)

Smirnov, 2018 - Smirnov, A.V. O formalizatsii umozaklyucheniya v protsessual'noi logike. Chast' II [Qiyās as a Formal Proof: the Way the Fuqahā' Argued. Part II], Filosofskii zhurnal [Philosophy Journal], 2018, Vol. 11, No. 1, pp. 5-27. (In Russian)

Volf, 2013 - Volf, M.N. Osnovaniya argumentatsii v rannegrecheskoi filosofii: analogiya kak tip argumentatsii [Foundations of Argumentation in Early Greek Philosophy: Analogy as a Type of Argumentation], Vestnik NGU. Filosofiya [Vestnik NSU], 2013, Vol. 11, No. 2, pp. 110-119. (In Russian)

Zaitseva, 2018 - Zaitseva, N.V. Kognitivno-fenomenologicheskaya interpretatsiya ritoricheskogo primera [Cognitive-Phenomeniological Interpretation of a Rhetorical Example], Vestnik Tomskogo gosudarstvennogo universiteta. Filosofiya. Sotsiologiya. Politologiya [Tomsk State University Journal of Philosophy, Sociology and Political Science], 2018, Vol. 45, pp. 14-24. (In Russian) 